\title{
Postinjury Inhibition of miR-181a Promotes Restoration of Hippocampal CA1 Neurons after Transient Forebrain Ischemia in Rats
}

\author{
[Drian B. Griffiths," Yi-Bing Ouyang," Lijun Xu, Xiaoyun Sun, Rona G. Giffard, and ${ }^{\circledR}$ Creed M. Stary
}

https://doi.org/10.1523/ENEURO.0002-19.2019

Department of Anesthesiology, Perioperative and Pain Medicine, Stanford University School of Medicine, Stanford, CA $94305-5117$

\begin{abstract}
The cellular and molecular mechanisms regulating postinjury neurogenesis in the adult hippocampus remain undefined. We have previously demonstrated that preinjury treatment with anti-microRNA (miR)-181a preserved neurons and prevented astrocyte dysfunction in the hippocampal cornu ammonis-1 (CA1) following transient forebrain ischemia. In the present study, we assessed postinjury treatment with anti-miR-181a on recovery of CA1 neurons following transient forebrain ischemia in rats. Stereotactic CA1 injection of miR-181a antagomir at either $2 \mathrm{~h}$ or $7 \mathrm{~d}$ postinjury resulted in improved restoration of CA1 measured at $28 \mathrm{~d}$ postinjury. Treatment with antagomir was associated with overexpression of the mir-181a target cell adhesion-associated, oncogenerelated protein and enhanced expression of the neuroprogenitor cell marker doublecortin (DCX) in the CA1. Assessment of $\mathrm{GFAP}^{+}$cell fate by Cre/Lox-mediated deletion demonstrated that some $\mathrm{GFAP}^{+}$cells in CA1 exhibited de novo DCX expression in response to injury. In vitro experiments using primary neuronal stem cells confirmed that miR-181a inhibition augmented the expression of DCX and directed cellular differentiation toward a neuronal fate. These results suggest that miR-181a inhibition plays a central role in the restoration of CA1 neurons via augmentation of early latent neurogenic gene activation in neural progenitor cells, including some reactive astrocytes. Therapeutic interventions targeting this restorative process may represent a novel postinjury approach to improve clinical outcomes in survivors of forebrain ischemia.
\end{abstract}

Key words: astrocytes; forebrain ischemia; hippocampus; microRNA; neurogenesis

\section{Significance Statement}

Persistent cognitive impairment is a major source of decreased quality of life for survivors of cardiac arrest, with impaired memory cited as the most severe long-term deficit (Moulaert et al., 2009). Interruptions in cerebral blood flow result in delayed death of hippocampal cornu ammonis-1 (CA1) neurons (Horn and Schlote, 1992). Pharmaceutical therapies given during this 2 week therapeutic window could drastically reduce the cognitive deficits experienced by survivors of forebrain ischemia. However, interventions that have attempted to directly target neurons after forebrain ischemia have so far failed to translate to effective therapies. Therefore, there is an urgent need for novel treatments that either provide protection against loss or hasten functional recovery of CA1 neurons.

Received January 2, 2019; accepted July 19, 2019; First published August 19, 2019.

The authors declare no competing financial interests.
Author contributions: B.B.G., Y.-B.O., L.X., and X.S. performed research; B.B.G., Y.-B.O., X.S., and C.M.S. analyzed data; B.B.G., Y.-B.O., and C.M.S. wrote the paper; Y.-B.O., R.G.G., and C.M.S. designed research. 


\section{Introduction}

In response to forebrain ischemia, the adult brain attempts to repair damage by producing new neurons not only in known neurogenic regions, but also in areas where neurogenesis does not normally occur, including the striatum (Magnusson et al., 2014) and hippocampal cornu ammonis-1 (CA1; Schmidt and Reymann, 2002; Bendel et al., 2005; Salazar-Colocho et al., 2008). In young adult rodent models of forebrain ischemia, neuronal recovery of CA1 is a well established phenomenon (Kirino, 1982); however, cell division is not a significant factor in observed neuronal recovery (Sugawara et al., 2000). In adult humans, delayed neuronal death is similarly a principle factor that contributes to impaired cognitive function following global cerebral ischemia (Horn and Schlote, 1992). However de novo neuronal proliferation and numbers of resident neuroprogenitor cells in the hippocampus drastically decrease after normal childhood brain development (Boldrini et al., 2018). Therefore, proliferative neurogenesis from resident neuroprogenitor cells cannot fully explain postinjury restoration of CA1 neurons after forebrain ischemia in adults.

Astrocytes, specialized glia that regulate neuronal homeostasis in health and injury, have been demonstrated to play a central role in neurogenesis in the adult brain (Cassé et al., 2018). We and others have provided increasing evidence of a protective role for astrocytes against ischemic injury in the adult (Ouyang et al., 2014; Stary and Giffard, 2015; Li and Stary, 2016). Astrocyte protection of CA1 neurons can be augmented by manipulation of endogenous expression of microRNAs (miRs), a class of small (19-22 nt) noncoding RNAs that regulate gene expression primarily at the post-transcriptional level. In particular, miR-181a is highly expressed in the adult brain after injury, and we have previously demonstrated that both pretreatment and post-treatment with miR-181a antagomir improves protection and recovery after focal cerebral ischemia (Ouyang et al., 2012b; Xu et al., 2015). In the setting of forebrain ischemia, we have previously demonstrated that miR-181a antagomir preinjury treatment preserves astrocyte function and protects CA1 neurons from delayed cell death (Moon et al., 2013). miR181a has been previously established to play a role in embryonic stem cell development of neurons through direct inhibition of cell adhesion-associated, oncogenerelated (CDON) protein expression, a critical regulator of embryonic neurogenesis (Gibert et al., 2014). Therefore, in the present study we did the following: (1) explored in vivo

This study was funded in part by American Heart Association Grant 18POST33990395 to B.B.G., National Institutes of Health Grants NS084396 and NS080177 to R.G.G., and American Heart Association Grant FTF19970029 to C.M.S.

${ }^{*}$ B.B.G. and Y.-B.O. contributed equally to this article.

Correspondence should be addressed to Brian B. Griffiths at Griffiths@ stanford.edu.

https://doi.org/10.1523/ENEURO.0002-19.2019

Copyright (C) 2019 Griffiths et al.

This is an open-access article distributed under the terms of the Creative Commons Attribution 4.0 International license, which permits unrestricted use, distribution and reproduction in any medium provided that the original work is properly attributed. the effect of postinjury anti-miR-181a treatment on the restoration of hippocampal CA1 neurons after forebrain ischemia, and the role astrocytes may play in this process; and, (2) assessed the effect of miR-181a in determining the neurogenic fate of stem cells in vitro.

\section{Materials and Methods}

\section{Forebrain ischemia}

All experimental protocols using animals were performed according to protocols approved by the Stanford University Animal Care and Use Committee and in accordance with the National Institutes of Health Guide for the Care and Use of Laboratory Animals. Forebrain ischemia was induced via two-vessel occlusion plus hypotension in adult (3 months of age; $300-400 \times g$ ), male Sprague Dawley rats (Charles River Laboratories), a model that reliably results in select destruction of CA1 neurons as described previously (Ouyang et al., 2007, 2012b). Briefly, hypotension (mean arterial pressure, $<40 \mathrm{mmHg}$ ) was induced by removing blood into heparinized sterile tubing during continuous femoral arterial blood pressure monitoring (Puritan Bennett). Carotid arteries were clamped bilaterally simultaneously for $10 \mathrm{~min}$. Rectal temperature $\left(37 \pm 0.5^{\circ} \mathrm{C}\right)$ was controlled by a homeothermic blanket (Harvard Apparatus). Respiratory rate, heart rate, and pulse oximetry were monitored with a small animal oximeter (STARR Life Sciences). After $10 \mathrm{~min}$, the clamps were removed and shed blood was reinfused.

\section{In vivo treatments}

Animals were randomly assigned to treatment group by coin flip to receive either stereotactic intraparenchymal injection with 5'6-FAM fluorescently labeled miR-181a-5p antagomir (3 pmol/g; Thermo Fisher Scientific) mixed 1:3 with the cationic lipid DOTAP (catalog \#11202375001, Roche Applied Science); or 5'6-FAM fluorescently labeled mismatch control (MM-con) treatment. The stereotactic injections were administered at either $2 \mathrm{~h}$ or $7 \mathrm{~d}$ after injury, and miR-181a antagomir or MM-con was stereotactically infused in the stratum moleculare of the CA1, allowing miR-181a or MM-con to perfuse into the CA1, CA3, and DG, as previously described (Ouyang et al., 2007, 2012b). See Fig. 3A for a visual representation. Animals were killed at 7, 14, 21, 28, 70, or $91 \mathrm{~d}$ after injury, and brains were fixed for histologic assessment.

\section{Fluorescent immunohistochemistry}

Ischemic or sham-operated rats were deeply anesthetized and transcardially perfused with cold $0.9 \%$ saline, followed by $4 \%$ paraformaldehyde (PFA) in PBS, $\mathrm{pH} 7.4$. Immunohistochemistry was performed as described previously (Xu et al., 2015). Briefly, brains were kept refrigerated in 4\% PFA in PBS, pH 7.4, for at least $3 \mathrm{~d}$ and then processed into $50 \mu \mathrm{m}$ sections with a vibratome (VT1000S, Leica Microsystems). Brain sections were blocked in serum overnight, incubated in primary antibodies (Table 1) overnight and then incubated in secondary antibodies overnight (Table 2). Immunofluorescent images of the marker for mature neuronal somas NeuN, the neuroprogenitor marker doublecortin (DCX), the astrocyte markers 
Table 1. Primary antibodies used for fluorescent immunohistochemistry

\begin{tabular}{llll}
\hline Protein & Host & \multicolumn{1}{c}{ Manufacturer } & Dilution \\
NeuN & Mouse & EMD Millipore, catalog \#MAB377; RRID:AB_2298772 & $1: 500$ \\
NeuN & Rabbit & Abcam, catalog \#ab177487; RRID:AB_2532109 & $1: 500$ \\
GFAP & Mouse & Abcam, catalog \#ab10062; RRID:AB_296804 & $1: 500$ \\
GFAP & Rabbit & Abcam, catalog \#ab5804; RRID:AB_2109645 & $1: 1000$ \\
GFAP & Chicken & Abcam, catalog \#ab4674; RRID:AB_304558 & $1: 500$ \\
DCX & Rabbit & Abcam, catalog \#ab18723; RRID:AB_732011 & $1: 1000$ \\
CDON & Mouse & Santa Cruz Biotechnology, catalog \#sC-377232 & $1: 500$ \\
MAP2 & Chicken & Abcam, catalog \#ab5392; RRID:AB_2138153 & $1: 500$ \\
\hline
\end{tabular}

glial fibrillary acidic protein (GFAP) and CDON (expressed in neuronal precursors) were acquired in hippocampal sections using an upright Zeiss Axio Imager M2 Multichannel Fluorescent Microscope. CA1 $\mathrm{NeuN}^{+}$cell populations were adjusted for total cell count using the nuclear stain $4^{\prime}, 6^{\prime}-$ diamidino-2-phenylindole dihydrochloride (DAPI; catalog \#10236276001, Sigma-Aldrich) and quantitated using 3D optical sectioning in the $z$-axis by structured illumination with an Apotome 2.0 using Stereolnvestigator software. The images presented are as close to representative as possible, although the conversion of $3 \mathrm{D}$ images to $2 \mathrm{D}$ may obscure some overlapping cells. Cellular colocalization was determined in 3D image stacks using Neurolucida version 17 software. For DCX and CDON staining analysis in the CA1 pyramidal layer, image stacks were analyzed using ImageJ by creating a threshold and measuring the mean illumination for each image section, collapsing each set of measurements to a single value for each animal.

\section{In vitro treatments}

Neural precursor cells were isolated postnatally from newborn mice at $24 \mathrm{~h}$. The brains were removed, freed of meninges, and diced with a sterile razor blade in dissociation buffer (DMEM; catalog \#D5796, Sigma-Aldrich) containing $2.5 \mathrm{U} / \mathrm{ml}$ papain (catalog \#1495005, Sigma-Aldrich), 250 $\mathrm{U} / \mathrm{ml}$ DNase I (catalog \#LS006330, Worthington Biochem), and 1 U/ml Dispase II (catalog \#04942078001, Roche Diagnostics). After a $1 \mathrm{~h}$ incubation at $37^{\circ}$, the cells were washed three times with DMEM supplemented with $10 \%$ fetal bovine serum (FBS; HyClone FBS; Cat \# SH3007103, Thermo Fisher Scientific). The cells were then resuspended in growth medium, Gibco Neurobasal A (catalog \#21103049, Thermo
Fisher Scientific), with 2 mM L-glutamine, $100 \mathrm{U} / \mathrm{ml}$ Gibco penicillin-streptomycin (catalog \#15140122, Thermo Fisher Scientific), $B_{27}$ without vitamin A (catalog \#A3353501, Thermo Fisher Scientific), $20 \mathrm{ng} / \mathrm{ml}$ fibroblast growth factor-2 (catalog \#100-18B, Peprotech), and $20 \mathrm{ng} / \mathrm{ml}$ epidermal growth factor (catalog \#AF-100-15, Peprotech); and plated at a density of 1 brain per six-well plate. Neural precursor cells proliferated and started to form neurospheres in 2-3 d, the plates were shaken every day to prevent attachment. Neural precursor cells were induced to differentiate by plating dissociated neurospheres in laminincoated 24-well plates, (the plates were coated with $10 \mu \mathrm{g} /$ well Gibco laminin (catalog \#23017015, Thermo Fisher Scientific) in distilled $\mathrm{H}_{2} \mathrm{O}\left(\mathrm{dH}_{2} \mathrm{O}\right)$ for 2-3 $\mathrm{h}$ and rinsed twice with $\mathrm{dH}_{2} \mathrm{O}$. The floating neurospheres were collected after passage through a $70 \mu \mathrm{m}$ cell strainer (catalog \#087712, Thermo Fisher Scientific) by centrifugation at $400 \times g$ for 5 min at $4^{\circ} \mathrm{C}$. The cell pellet was gently triturated with a $200 \mu \mathrm{l}$ pipette tip 15-20 times, resuspended in a small volume of differentiation medium, Neurobasal A, B27, 1\% FBS, 1 $\mathrm{ng} / \mathrm{ml}$ fibroblast growth factor-2, $10 \mathrm{ng} / \mathrm{ml}$ brain-derived neurotrophic factor (catalog \#450-02, Peprotech), and 10 $\mathrm{ng} / \mathrm{ml}$ neurotrophic factor-3 (catalog \#450-03; Peprotech). The cells were plated onto laminin-coated 24-well plates at a density of 200,000 cells $/ \mathrm{ml}$. One-half of the differentiation medium was changed every $2-3 \mathrm{~d}$. Cells were grown in $21 \%$ $\mathrm{O}_{2} / 5 \% \mathrm{CO}_{2}$ and were allowed to differentiate for different times dependent on the experimental design as detailed previously (Sun et al., 2015). Transfection started on day 1 after cells were grown in differentiation medium. To transfect cells, 50 pmol miR-181a-5p mimic or inhibitor was used with FuGENE HD Reagent (catalog \#E2311, Promega) every $3 \mathrm{~d}$,

Table 2. Secondary antibodies used for fluorescent immunohistochemistry

\begin{tabular}{|c|c|c|}
\hline Fluorophore & Host & Manufacturer \\
\hline Alexa Fluor 488 & Donkey anti-rabbit IgG & $\begin{array}{l}\text { Thermo Fisher Scientific, catalog \#A21206 } \\
\text { RRID:AB_2535792 }\end{array}$ \\
\hline Alexa Fluor 488 & Donkey anti-goat lgG & $\begin{array}{l}\text { Thermo Fisher Scientific, catalog \#A11055 } \\
\text { RRID:AB_2534102 }\end{array}$ \\
\hline Alexa Fluor 488 & Goat anti-chicken lgG & $\begin{array}{l}\text { Thermo Fisher Scientific, catalog \#A32931 } \\
\text { RRID:AB_2340375 }\end{array}$ \\
\hline Alexa Fluor 594 & Donkey anti-goat IgG & $\begin{array}{l}\text { Thermo Fisher Scientific, catalog \#A11058 } \\
\text { RRID:AB_2534105 }\end{array}$ \\
\hline Alexa Fluor 594 & Donkey anti-sheep lgG & $\begin{array}{l}\text { Thermo Fisher Scientific, catalog \#A11016 } \\
\text { RRID:AB_2534083 }\end{array}$ \\
\hline Alexa Fluor 594 & Donkey anti-rabbit IgG & $\begin{array}{l}\text { Thermo Fisher Scientific, catalog \#SA5-1005 } \\
\text { RRID:AB_2556620 }\end{array}$ \\
\hline
\end{tabular}


Table 3. Primers for cloning fate-mapping plasmid promoter sequences

\begin{tabular}{ll}
\hline Promoter & \multicolumn{1}{c}{ PCR Primers } \\
DCX & CTCGAGATATTCTTATCGCCGCACATC \\
GFAP & GCTAGCTTGGTGGAACCACAGCAACCTGA \\
& AAGCTTACGTAGCGTGGTTTAC
\end{tabular}

and the transfection reagent was washed out after overnight incubation. All cell culture experiments were repeated in triplicate.

\section{Immunocytochemistry}

Fluorescence immunocytochemistry was assessed in cell cultures grown in 24-well plates. The cultures were washed with PBS and then fixed in 4\% PFA for $30 \mathrm{~min}$ at room temperature. The cells were then washed three times with PBS, and nonspecific binding was blocked with $5 \%$ normal horse serum and $0.1 \%$ Triton $X-100$ in PBS for $1 \mathrm{~h}$. The cells were subsequently incubated with primary antibodies diluted in blocking buffer overnight at $4^{\circ} \mathrm{C}$ (Table 1). Cells were washed three times in PBS plus $0.1 \%$ Triton X-100 buffer and subsequently incubated with the appropriate secondary antibodies (Table 2). Cell nuclei were counterstained with DAPI $(0.5 \mu \mathrm{g} / \mathrm{ml}$; SigmaAldrich). To eliminate observer selection bias, immunofluorescent images (nine per well) were acquired using an automated epifluorescence microscope (LS720, Etaluma; Lumaview software version 17.11, Etaluma). Fluorescence intensity was then quantified using Fiji (version $1.51 \mathrm{~s})$ by an observer blinded to conditions.

\section{Fate mapping DCX/GFAP Cre-Lox system}

We designed a DCX/GFAP plasmid to track de novo DCX expression in GFAP-producing cells. The plasmid contained a DCX promoter linked to both CRE and mCherry expression, and a floxed GFAP promoter-EGFP sequence. In the absence of DCX expression, EGFP (green) is expressed from a GFAP promoter in transfected astrocytes, which express GFAP. On activation of the DCX promoter, Cre and mCherry (red) are coexpressed. Cre removes the floxed GFAP promoter-EGFP sequence and EGFP expression is terminated, while $\mathrm{DCX}^{+}$cells express mCherry. Cells expressing only EGFP lack DCX expression, but any $\mathrm{EGFP}^{+} / \mathrm{mCherry}^{+}$cells indicate de novo expression of $\mathrm{DCX}$ in a $\mathrm{GFAP}^{+}$cell. DCX (Voloboueva et al., 2010) and GFAP (Xu et al., 2010) promoters were obtained from prior clones (listed in Table 3). The plasmid was sterotactically injected $24 \mathrm{~h}$ before ischemia, and animals were killed $7 \mathrm{~d}$ after ischemia and treated as detailed in the immunohistochemistry methods above.

\section{Statistical analysis}

The numbers of animals per group are indicated in the figure legends. Data are reported as the mean \pm SEM. For cell culture experiments, technical replicates (at least four wells) were averaged and treated as a single sample, and all experiments were repeated in triplicate (three separate dissections). All statistical analyses were performed on
SPSS (version 22, IMB; RRID:SCR_002865). Statistical difference was determined using Student's $t$ test for comparison of two groups with Cohen's d as a measure of effect size, or ANOVA for experiments with more than two groups with partial $\eta^{2}$ as a measure of effect size with Tukey's post hoc test for group comparisons. In all analyses, $p<0.05$ was considered statistically significant.

\section{Results}

\section{Loss and restoration of hippocampal CA1 neurons following forebrain ischemia}

An established characteristic of the injury model used in the present study is delayed, extensive loss of hippocampal CA1 pyramidal neurons (Pulsinelli et al., 1982). Immunostaining for NeuN, a marker for mature neurons, confirmed the expected loss of CA1 neurons after forebrain ischemia (Fig. $1 A ; F_{(5,15)}=315.24, p<0.001$, partial $\eta^{2}=0.99$ ). Post hoc analysis indicated that neuronal loss had occurred by $7 \mathrm{~d}$ after ischemia with the number of CA1 neurons dropping to $\sim 38 \%$ compared with sham controls. By $14 \mathrm{~d}$ after ischemia, the CA1 neuronal population was almost completely abolished with only $\sim 6 \%$ of $\mathrm{NeuN}^{+}$cells remaining compared with sham animals (Fig. 1B). Significant $(p<0.01)$ recovery of mature neurons was observed by $21 \mathrm{~d}$ (Fig. 1C) relative to the $14 \mathrm{~d}$ nadir, and continued to increase to a plateau of $\sim 41 \%$ of the original number by $70 \mathrm{~d}$, after which the number of $\mathrm{NeuN}^{+}$neurons remained mostly unchanged by $90 \mathrm{~d}$ postinjury (Fig. 1B,C).

\section{Reactive astrocytes were induced after forebrain ischemia}

In conjunction with the reappearance of mature neurons after forebrain ischemia, hippocampal $\mathrm{GFAP}^{+}$astrocytes were quantitated via immunohistochemical labeling. In sham control animals, $\mathrm{GFAP}^{+}$cells in CA1 were not localized to the pyramidal neuronal layer. Following forebrain ischemia, we observed a significant increase of $\mathrm{GFAP}^{+}$cells in the CA1 pyramidal layer compared with sham controls by $7 \mathrm{~d}$ postinjury, which was sustained until 70 d postinjury $\left(F_{(5,33)}=42.55, p=<0.001\right.$, partial $\eta^{2}=$ 0.87 ; Fig. 2A). Post hoc analysis revealed that the number of $\mathrm{GFAP}^{+}$cells peaked at $14 \mathrm{~d}$ postinjury, $7 \mathrm{~d}$ before when CA1 first demonstrated observable recovery of mature neurons (Fig. 2B). By $91 \mathrm{~d}$, the total number of GFAP ${ }^{+}$ cells in hippocampus had largely returned to the preinjury baseline (Fig. 2C). To examine whether postinjury miR181a inhibition enhanced the recovery process, we treated animals with antagomir either immediately $(2 \mathrm{~h})$ after injury or $7 \mathrm{~d}$ after injury, time points that precede both maximum cell death $(14 \mathrm{~d}$; Fig. $1 D)$ and the maximum number of reactive astrocytes in the CA1 (14 d; Fig. 2B).

\section{Enhanced recovery of hippocampal CA1 neurons by miR-181a antagomir}

Stereotactic injection of fluorescently labeled miR-181a antagomir exhibited robust transduction throughout CA1 and DG in the ipsilateral hippocampus (Fig. $3 A$ ) but was completely absent in the contralateral hippocampus. For all experiments, only the ipsilateral side of the hippocam- 
A

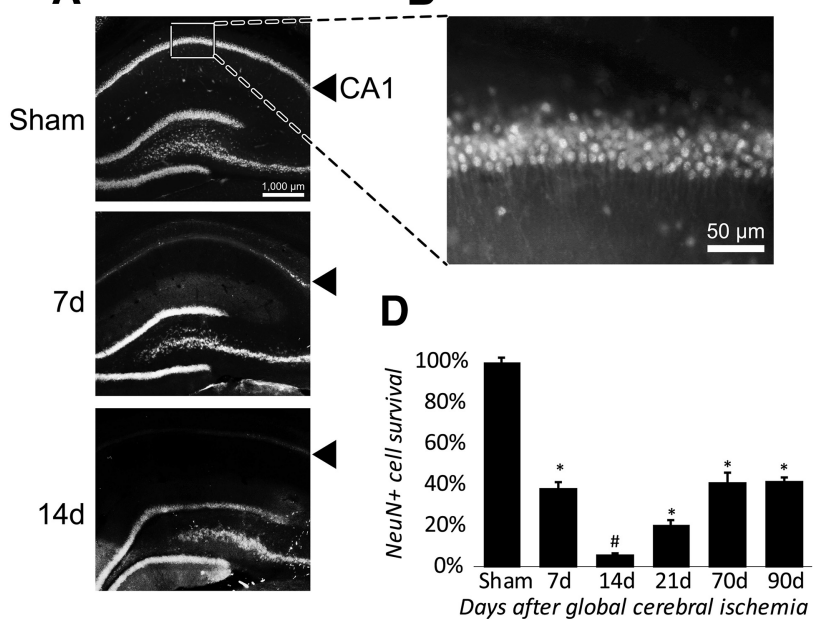

C
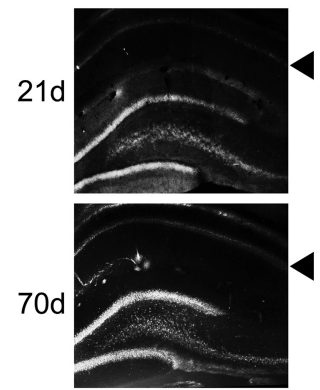

$90 \mathrm{~d}$

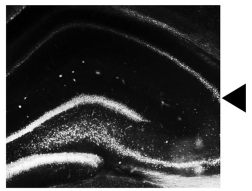

Figure 1. CA1 neuronal loss and recovery following forebrain ischemia. $\boldsymbol{A}$, Forebrain ischemia results in delayed cell death of mature $\left(\mathrm{NeuN}^{+}\right.$) neurons in hippocampal CA1 (denoted by solid arrowhead). B, Magnified view of CA1 neurons in sham animals. $\boldsymbol{C}$, Gradual and partial postinjury recovery of $\mathrm{NeuN}^{+}$neurons in CA1. $\boldsymbol{D}$, Quantitative measurement of postinjury loss and recovery of CA1 neurons. $N=3-6 /$ group, $* p<0.05$ compared with sham, $\# p<0.05$ compared with every group. Error bars are mean \pm SEM.

pus that received either the MM-con of miR-181a inhibitor treatment was analyzed. There were no differences between $2 \mathrm{~h}$ and $7 \mathrm{~d}$ MM-con-treated animals. MiR-181a antagomir significantly increased the survival of mature neurons in CA1 $14 \mathrm{~d}$ after ischemia when injected $2 \mathrm{~h}$ or $7 \mathrm{~d}$ postinjury (Fig. 3B,C; $F_{(2,20)}=124.76, p<0.001$, partial $\left.\eta^{2}=0.93\right)$. Interestingly, both the $2 \mathrm{~h}$ and $7 \mathrm{~d}$ injection exhibited enhanced recovery of CA1 neurons relative to controls when assessed $28 \mathrm{~d}$ after ischemia, though the animals receiving the $2 \mathrm{~h}$ injection demonstrated slightly greater recovery than those receiving the $7 \mathrm{~d}$ injection (Fig. $3 D, E ; F_{(2,22)}=456.36, p<0.001$,
A
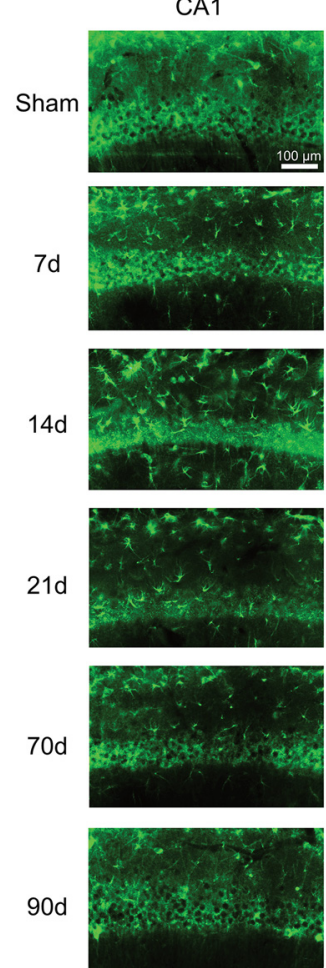

B

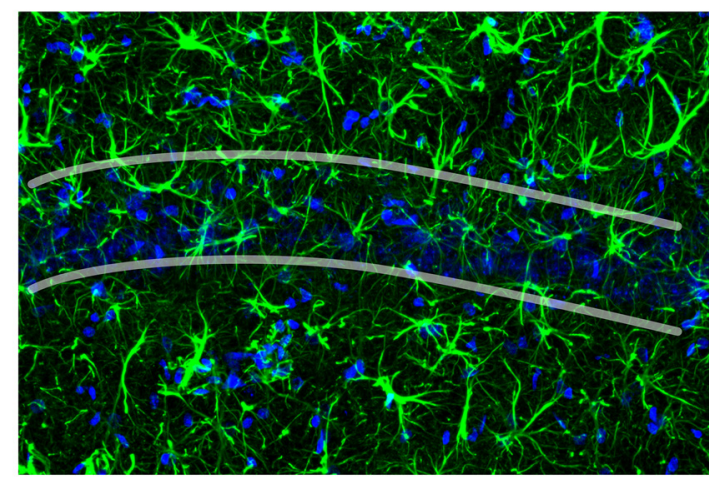

C

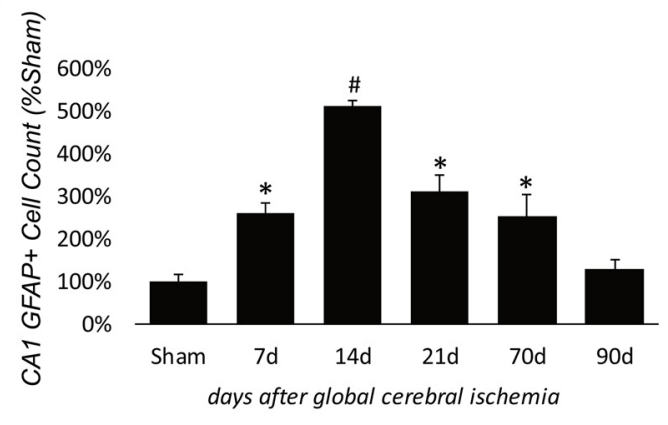

Figure 2. Hippocampal increased GFAP expression in the CA1 pyramidal layer following forebrain ischemia. $\boldsymbol{A}$, Fluorescent labeling of GFAP after forebrain ischemia in the hippocampus ( $50 \times$ magnification). $\boldsymbol{B}$, Enlarged $(200 \times)$ example of CA1 pyramidal layer at 14 $\mathrm{d}$ after forebrain ischemia. $\boldsymbol{C}$, Postinjury quantification of GFAP ${ }^{+}$cells in CA1. $N=3-6 /$ group, $* p<0.05$ compared with sham, \#p $<$ 0.05 compared with every group. Error bars are mean \pm SEM. 
A
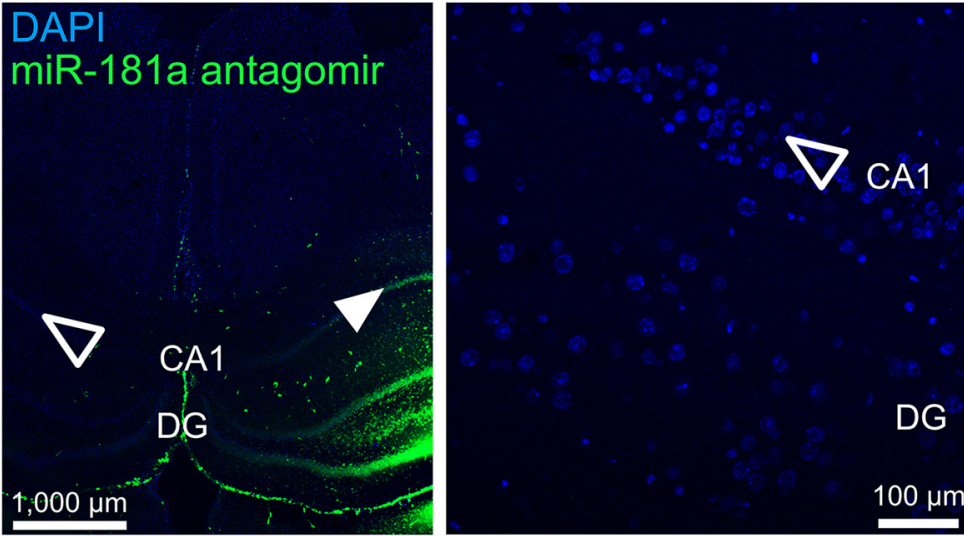

Contralateral

B

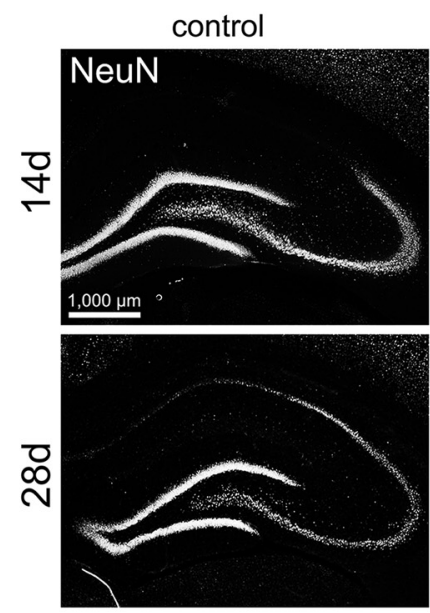

control

F

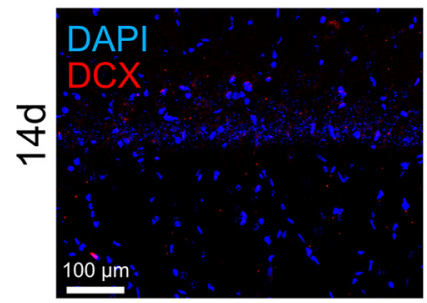

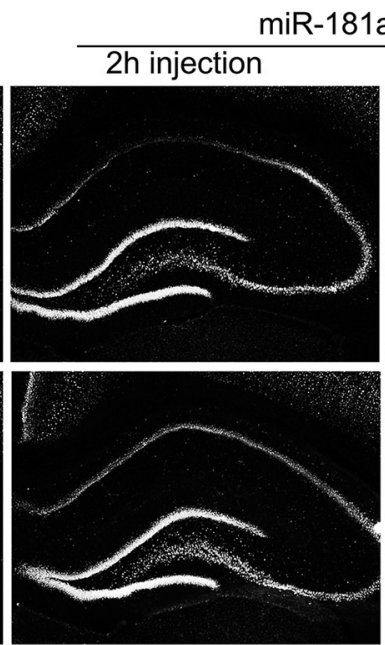

\section{7d injection}

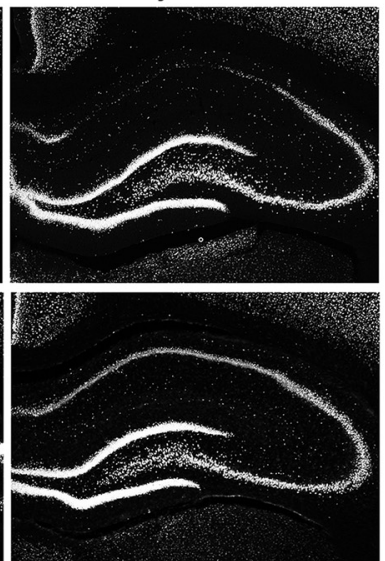

miR-181a inhibitor

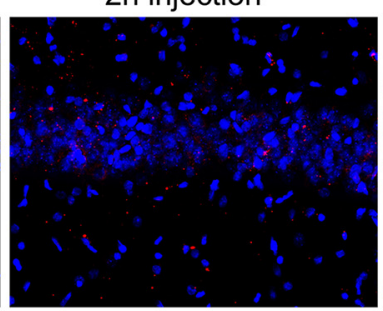

$7 d$ injection

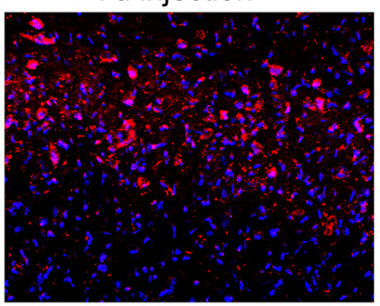

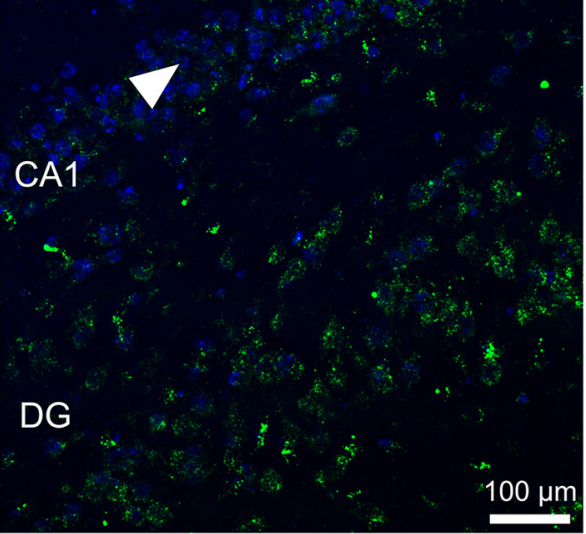

Ipsilateral

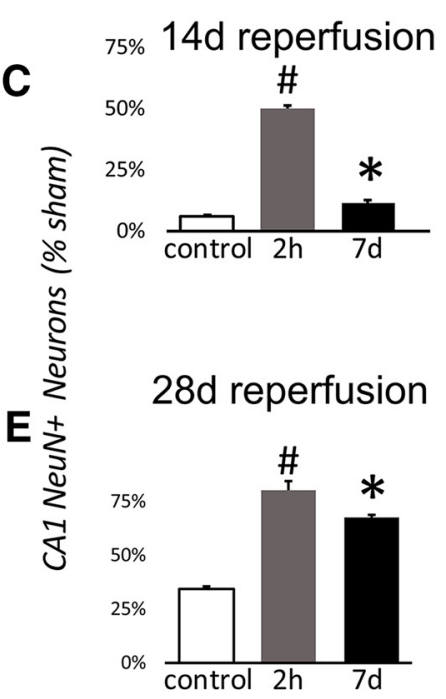

\section{$14 d$ reperfusion}

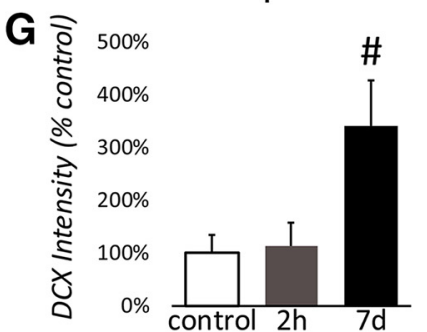

Figure 3. Effect of postinjury miR-181a antagomir treatment on CA1 NeuN and DCX precursor expression and long-term CA1 neuronal recovery following forebrain ischemia. $\boldsymbol{A}$, Fluorescently labeled (green, 6-FAM) miR-181a antagomir is detected in ipsilateral hippocampus (solid arrowhead), but not in contralaterally injected hippocampus (empty arrowhead). Magnified views of contralateral and ipsilateral CA1 following injection of 6-FAM-labeled miR-181a antagomir. $\boldsymbol{B}, \boldsymbol{C}$, Examples $(\boldsymbol{B})$ and quantification $(\boldsymbol{C})$ of ipsilateral hippocampal NeuN fluorescent labeling $14 \mathrm{~d}$ after forebrain ischemia in postinjury MM-con-treated and antagomir-treated animals. $\boldsymbol{D}, \boldsymbol{E}$, Examples $(\boldsymbol{D})$ and quantification $(\boldsymbol{E})$ of ipsilateral hippocampal NeuN fluorescent labeling at $28 \mathrm{~d}$ postinjury in MM-con-treated and antagomir-treated animals. $\boldsymbol{F}, \boldsymbol{G}$, Examples $(\boldsymbol{F})$ and quantification $(\boldsymbol{G})$ of ipsilateral CA1 DCX ${ }^{+}$cells $14 \mathrm{~d}$ after forebrain ischemia with MM-con or antagomir treatment $2 \mathrm{~h}$ or $7 \mathrm{~d}$ after forebrain ischemia. $N=5-6$ for each condition. $* p<0.05$ compared with sham, $\# p<0.05$ compared with every group. Error bars are mean \pm SEM.

partial $\left.\eta^{2}=0.98\right)$. Further inspection of the CA1 revealed that animals injected with miR-181a antagomir at $7 \mathrm{~d}$ after ischemia had significantly greater levels of DCX fluorescence in the CA1 pyramidal layer than those treated at $2 \mathrm{~h}$ (Fig. $3 F, G ; t_{(7)}=2.50, p=0.041$, Cohen's $d=3.32$ ).

\section{Promotion of neurogenesis by miR-181a antagomir} through CDON overexpression

The embryonic regulator of neurogenesis, CDON, has been previously identified as a target of miR-181a (Gibert et al., 2014). In the adult rats used in the present study, CDON expression was largely undetectable $14 \mathrm{~d}$ following 
A

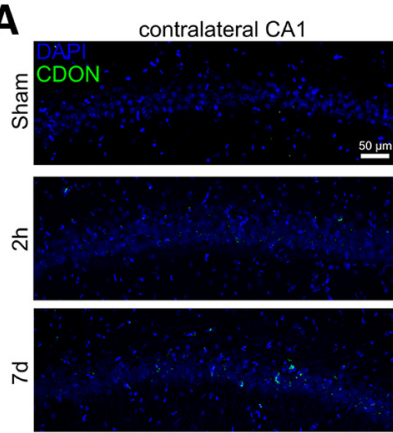

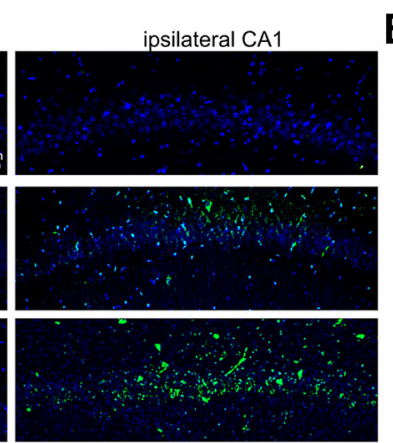

B

Figure 4. Postinjury CDON expression in CA1 following forebrain ischemia. $\boldsymbol{A}$, Immunofluorescent labeling of CDON expression (green) and all nuclei (DAPI) in CA1 $14 \mathrm{~d}$ after forebrain ischemia with MM control treatment or $2 \mathrm{~h}$ or $7 \mathrm{~d}$ postinjury antagomir treatment. B, Quantification of CDON in CA1 $14 \mathrm{~d}$ after forebrain ischemia with the three treatment groups. $N=4$ for each condition, $* p<0.05$ difference versus sham. Error bars are mean \pm SEM. n.s. $=$ not significant.

sham surgery (data not shown) and was only minimally detected after forebrain ischemia in treated MM-con animals (Fig 4A). Treatment with miR-181a antagomir $7 \mathrm{~d}$ postinjury resulted in a significant increase in hippocampal CDON expression in the CA1 pyramidal layer (Fig. 4B; $t_{(7)}=-2.45, p=0.040$, Cohen's $\left.d=-1.61\right)$. MiR-181a antagomir treatment at $2 \mathrm{~d}$ postinjury demonstrated a similar trend, though it failed to reach significance $(p=$ 0.161).

\section{Coexpression of DCX with GFAP after forebrain ischemia}

We see an increase in reactive astrocytes localized to the CA1 pyramidal cell layer at $14 \mathrm{~d}$, and prior observations in adult animals have described de novo conversion of astrocytes to neuronal precursors cells following injury (Buffo et al., 2008; Gabel et al., 2016). We investigated whether this phenomenon could also account for CA1 neuronal recovery following forebrain ischemia. Immature neurons have been previously observed to coexpress GFAP in adult neurogenesis (Garcia et al., 2004), while mature astrocytes have been demonstrated to have the capacity to dedifferentiate and express DCX (Verwer et al., 2007). To differentiate between these two possible origins for $\mathrm{DCX}^{+}$cells, we constructed a DCX/GFAP CreLox fate-mapping plasmid (Fig. 5A). The $24 \mathrm{~h}$ following stereotactic injection of the plasmid sham animals displayed only EGFP (green) fluorescence in CA1 pyramidal layer (Fig. 5B,C), indicating successful transduction and reporting in astrocytes, but a lack of DCX activity in these $\mathrm{GFAP}^{+}$cells. In contrast, forebrain ischemia after plasmid treatment resulted in robust expression of mCherry (red), indicating activation of DCX transcription, with substantial coexpression with EGFP (Fig. 5D,E). These observations suggest that at least a subset of $\mathrm{DCX}^{+}$cells represented astrocytes that began to express DCX de novo in response to injury.

\section{miR-181a inhibition promotes neurogenesis in neural stem cells}

To test the hypothesis that miR-181a contributes to the regulation of cellular differentiation, we altered miR-181a levels in growing neural stem cell cultures. By day in vitro 4 (DIV4), cells with suppressed miR-181a expression due to treatment with inhibitor demonstrated a marked increase in the number of $\mathrm{DCX}^{+}$cells compared with cells treated with miR-181a mimic or controls (Fig. $6 A, B ; F_{(2,26)}$ $=7.54, p=0.003$, partial $\left.\eta^{2}=0.37\right)$. Consistent with early elevations in DCX, cells assessed later at DIV10 demonstrated significantly greater expression of the mature neuronal marker MAP2 (microtubule associated protein 2) if miR-181a was suppressed with inhibitor compared with cells treated with mimic (Fig. 6C,D; $F_{(2,27)}=8.20, p=$ 0.002 , partial $\left.\eta^{2}=0.38\right)$. Qualitatively, immunocytochemical MAP2 staining also revealed morphologic differences in the mature neuronal population when miR-181a levels were altered: miR-181a inhibitor treatment tended to generate larger neurons with longer processes, versus treatment with mimic (Fig. 6E,F). The numbers of cells expressing the mature astrocyte marker GFAP were unaffected by miR-181a inhibitor or mimic (data not shown).

\section{Discussion}

In the present study, we report that postinjury treatment with miR-181a antagomir at $2 \mathrm{~h}$ or $7 \mathrm{~d}$ increased the hippocampal CA1 neuronal count $28 \mathrm{~d}$ after forebrain ischemia. One difference between the two treatments was that $2 \mathrm{~h}$ postinjury treatment might be considered neuroprotective and restorative, while $7 \mathrm{~d}$ postinjury treatment was only restorative. Within the window of postinjury neuroinflammation, $2 \mathrm{~h}$ postinjury treatment likely prevented some of the initial loss of CA1 neurons, since CA1 neuronal cell counts did not fall as far when assessed on day 14, the nadir for CA1 neuronal loss. miR-181a inhibition has been shown to be protective in several other brain injury models, such as focal ischemia (Ouyang et al., 2012a; Xu et al., 2015; Stary et al., 2017), epilepsy (Ren et al., 2016), and Parkinson's disease (Hegarty et al., 2018). Notably, miR-181a inhibition has been demonstrated to decrease inflammation (Xie et al., 2013a), and miR-181a is expressed early during the inflammatory response, but at lower levels in the chronic postinjury phase (Xie et al., 2013b). At 7 d postinjury, any acute inflammation from reperfusion injury would have subsided when CA1 neuronal loss already approached 60\% (Fig. 1). Prevention from additional loss could not alone account for the observed difference at $28 \mathrm{~d}$ postinjury. Instead, our observations suggest that miR-181a inhibition instead 
A

pDCX-cre-mCherry-GFAP-GFP

8810 bp

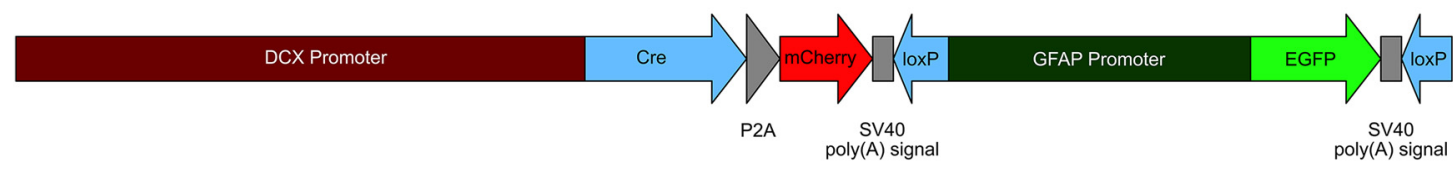

B

C
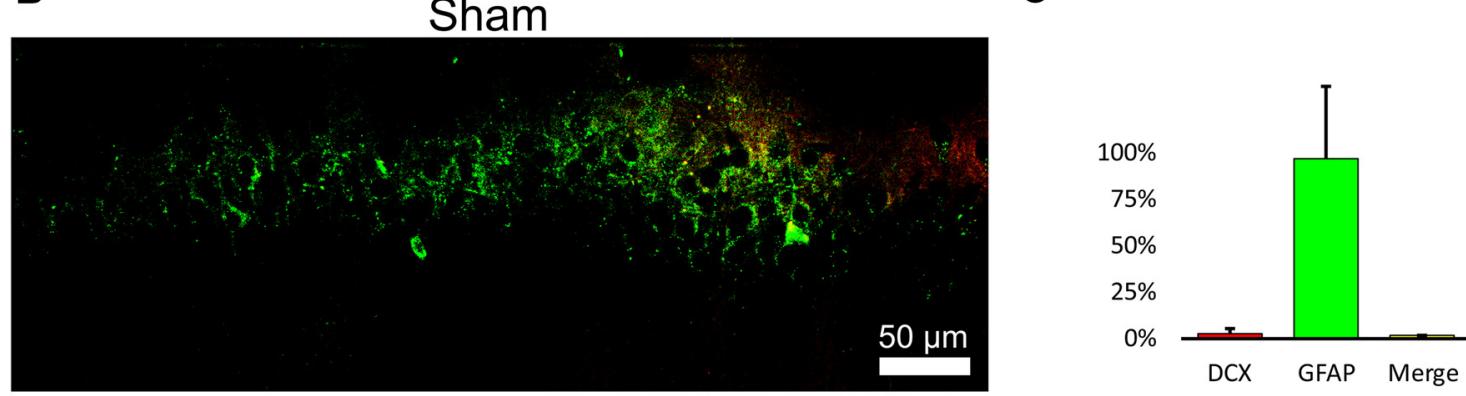

D

Forebrain Ischemia

E
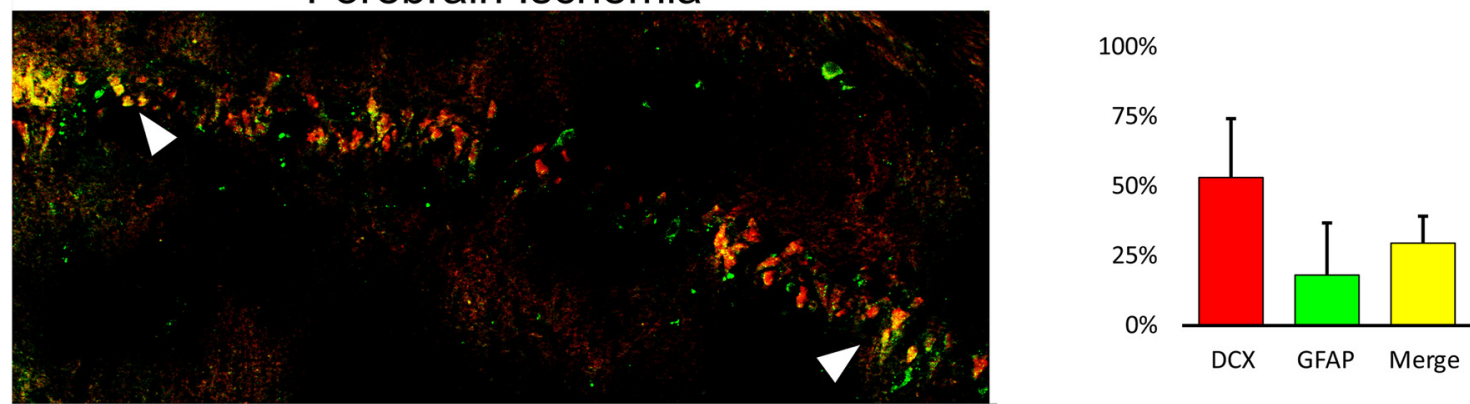

Figure 5. Fate-mapping postinjury DCX expression following forebrain ischemia. $\boldsymbol{A}$, Diagram and map of Cre-Lox GFAP/DCX fate-mapping plasmid. A floxed GFAP promoter EGFP (green) reporter and a DCX promoter controlling Cre and mCherry (red) reporter are included on the same plasmid. In the absence of DCX activity, astrocytes fluoresce green. With DCX activation, mCherry is expressed and EGFP expression is simultaneously terminated. $\boldsymbol{B}, \boldsymbol{C}$, Sham animals display very little new EGFP expression in CA1 after transfection, and no mCherry. $\boldsymbol{D}, \boldsymbol{E}$, Following forebrain ischemia $(7 \mathrm{~d})$, colocalization of EGFP/c-Tomato is evident in CA1, suggesting de novo expression of DCX in GFAP-expressing cells. The graphs indicate relative expression of $\mathrm{DCX}^{+}$only, GFAP ${ }^{+}$only, and DCX/GFAP ${ }^{+}$double-positive cells. Error bars are mean \pm SEM.

augmented endogenous activation of local pluripotent cells and their development into $\mathrm{NeuN}^{+}$neurons, at least in part originating from dedifferentiated astrocytes (Robel et al., 2011).

In the present study, we observed that $14 \mathrm{~d}$ postinjury coincided with both the peak of CA1 neuronal cell loss and the peak of GFAP expression (Figs. 1,2). A similar time course has been observed in other brain injury models whereby latent activation of neurogenic pathways in astrocytes was observed as early as 1 week postinjury (Simon et al., 2011). Distinct from proliferative neurogenesis, several models have demonstrated the potential for astrocytes to dedifferentiate and reprogram into neurons in response to injury (Buffo et al., 2008; Robel et al., 2011; Magnusson et al., 2014). Prior studies have demonstrated that CA1 neurogenesis is not due to cell division, supported by observations of minimal BrdU labeling during the restorative phase in CA1 pyramidal layers (Sugawara et al., 2000). In the absence of proliferating or migrating neuroprogenitors as a source of new neurons in CA1, the activation of pre-existing localized cells remains a possi- bility. Neural progenitors are not prevalent in the CA1, but astrocytes are common (Zeisel et al., 2015). We observed DCX expression in GFAP ${ }^{+}$cells in postinjury CA1 (Fig. 5), suggesting that astrocytes were not directly transdifferentiating, but instead that some may be dedifferentiating secondary to de novo expression of latent neuroprogenitor genes. Neurogenic properties of astrocytes appear to be dependent on local environmental factors, including Notch signaling (Imayoshi et al., 2008; Magnusson et al., 2014) and CDON expression. CDON is normally silenced through interactions with MeCP2 in astrocytes (Yasui et al., 2013); however, in the present study we observed a significant postinjury (14 d) increase in CDON expression (Fig. 4) in animals treated with miR-181a antagomir $7 \mathrm{~d}$ postinjury, but not in animals treated $2 \mathrm{~h}$ postinjury. It is not unreasonable to expect a residual effect on neurogenesis in CA1 with injection at $2 \mathrm{~h}$ postinjury, but this does not appear to be the primary mechanism.

Recent reviews have focused on specialized astrocytes that function as neural stem cells in the adult brain (Götz et al., 2015; Magnusson and Frisén, 2016; Platel and 

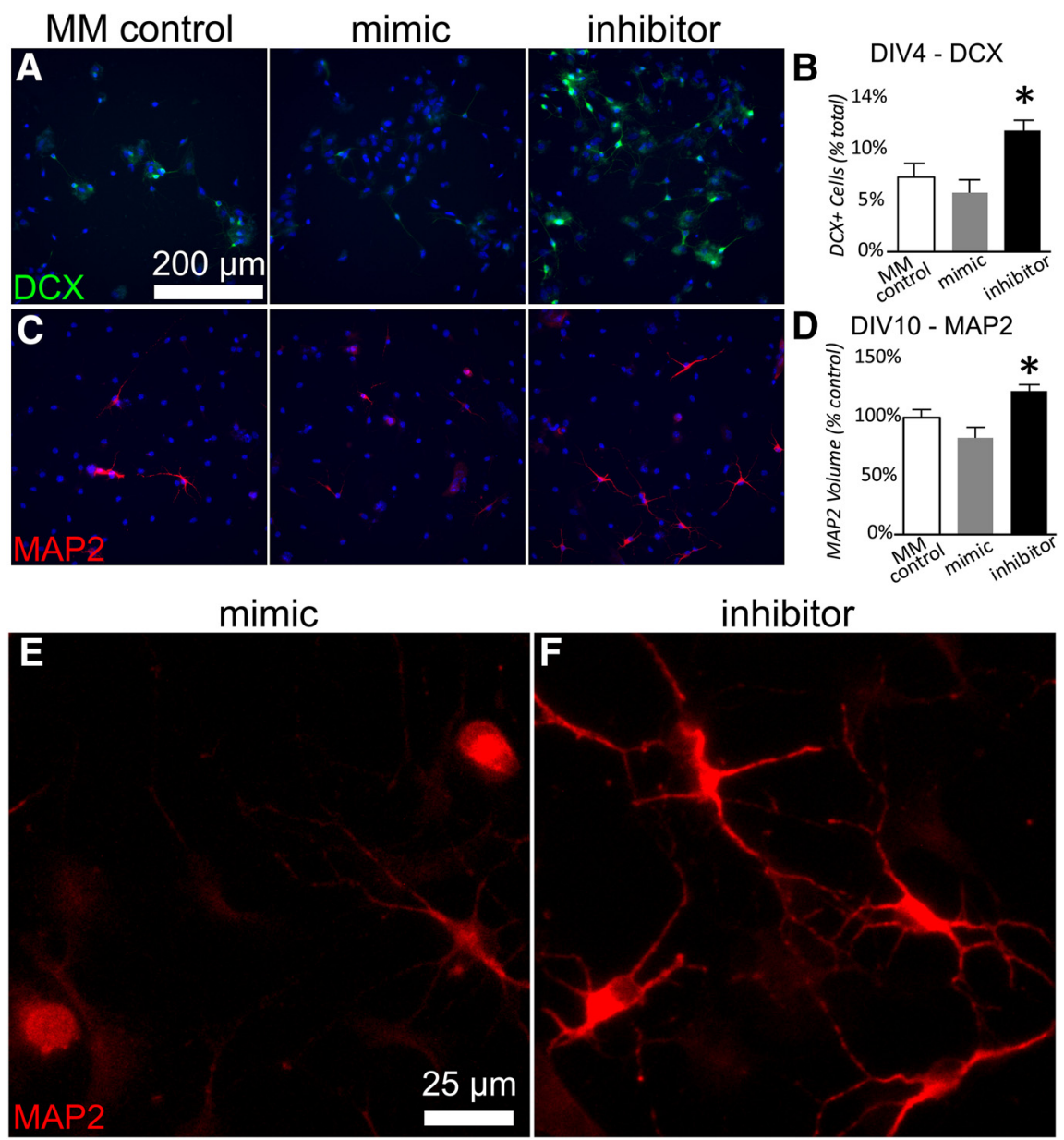

Figure 6. Effect of miR-181a in neural stem cell differentiation. $\boldsymbol{A}, \boldsymbol{B}$, Examples $(\boldsymbol{A})$ and quantification $(\boldsymbol{B})$ of DCX expression at DIV4 in neural stem cells treated with miR-181a inhibitor, mimic, or MM control. $\boldsymbol{C}, \boldsymbol{B}$, Examples $(\boldsymbol{C})$ and quantification $(\boldsymbol{D})$ of MAP2 expression at DIV10 in neuronal stem cells treated with miR-181a inhibitor, mimic, or MM control. Ten images were averaged for each sample well, then a grand average was generated for each condition. Experiments were repeated three times. The reported means represent the intracondition averages for the three experiments, $N=3$ for each condition. Error bars are sample mean \pm SEM. $* p$ $<0.05$ versus all other groups. $\boldsymbol{E}, \boldsymbol{F}$, Enlarged $(400 \times)$ images of MAP2 ${ }^{+}$cells treated with miR-181a mimic $(\boldsymbol{E})$ or inhibitor $(\boldsymbol{F})$ reflecting qualitative differences in neuronal morphology between treatments.

Bordey, 2016). After distal permanent middle cerebral artery occlusion surgery, self-renewal, and differentiation of reactive astrocyte-derived neural stem/progenitor cells were described, isolated from the cortical peri-infarct area (Shimada et al., 2012). Following focal cerebral ischemia in rodents, thousands of newborn neuroblasts appear in the striatum over the following weeks and months (Arvidsson et al., 2002; Li and Clevers, 2010; Magnusson et al., 2014), and one-third to two-thirds of newborn neurons are estimated to have been generated by local astrocytes (Magnusson et al., 2014). The expression of miR-181a decreases in neural progenitor cells in the subventricular zone after stroke (Liu et al., 2017), a time of increased neurogenesis, further suggesting that miR-181a impedes neuronal differentiation, a hypothesis supported by our observations of increased MAP2 expression in stem cells treated with miR-181a inhibitor (Fig. 6). Unregulated expression of miR-181a is seen in many malignant cancers (Feng et al., 2018), suggesting some level of control over cell fate. Additionally, MAP2 ${ }^{+}$cells treated with miR-181a inhib- itor exhibit altered cell morphology due to the known interactions of the inhibitor with cell fate regulators, but do not stain positive for GFAP, providing additional evidence that they are neurons and not astrocytes. Further studies should reveal whether new neurons born in the presence of miR181a inhibitor have altered function or assume a normal phenotype when the influence of the inhibitor is removed.

It is also likely that restored neurons in CA1 are derived from another cell type other than astrocytes. Microglia and oligodendrocytes are both prevalent throughout the hippocampus (Zeisel et al., 2015), and have also been demonstrated to have the capacity to dedifferentiate (Grinspan et al., 1996; Yokoyama et al., 2004). Additional experiments should investigate which other cell types in the brain contribute to this phenomenon using genetic fate mapping. Another limitation of the present study is that the analysis of DCX expression and CA1 recovery were performed at 1 week time intervals. Performing the analysis with shorter intervals may reveal a more comprehensive time course of dedifferentiating cells and better 
define the postinjury therapeutic window for survivors of forebrain ischemia.

In summary, hippocampal CA1 has an endogenous capacity to restore neurons in response to forebrain ischemia. Our observations suggest that this process can be augmented by postinjury treatment with anti-miR-181a. Cellular colocalization and fate mapping further suggest that this process may be mediated by the activation of latent neurogenic pathways in astrocytes. We confirmed in vitro that miR-181a inhibition has the capacity to direct stem cells toward a neuronal fate. Future investigations should extend these findings to functional assays to assess the role of miR-181a in cognitive recovery from forebrain ischemia, and in recovery from other models of injury in the adult brain.

\section{References}

Arvidsson A, Collin T, Kirik D, Kokaia Z, Lindvall O (2002) Neuronal replacement from endogenous precursors in the adult brain after stroke. Nat Med 8:963970.

Bendel O, Bueters T, von Euler M, Ögren SO, Sandin J, von Euler G (2005) Reappearance of hippocampal CA1 neurons after ischemia is associated with recovery of learning and memory. $\mathrm{J}$ Cereb Blood Flow Metab 25:1586-1595.

Boldrini M, Fulmore CA, Tartt AN, Simeon LR, Pavlova I, Poposka V, Rosoklija GB, Stankov A, Arango V, Dwork AJ, Hen R, Mann JJ (2018) Human hippocampal neurogenesis persists throughout aging. Cell Stem Cell 22:589-599.e5.

Buffo A, Rite I, Tripathi P, Lepier A, Colak D, Horn A-P, Mori T, Götz $M(2008)$ Origin and progeny of reactive gliosis: a source of multipotent cells in the injured brain. Proc Natl Acad Sci U S A 105:3581-3586.

Cassé F, Richetin K, Toni N (2018) Astrocytes' contribution to adult neurogenesis in physiology and Alzheimer's disease. Front Cell Neurosci 12:432.

Feng X, Zhang C, Yang Y, Hou D, Zhu A (2018) Role of miR-181a in the process of apoptosis of multiple malignant tumors: a literature review. Adv Clin Exp Med 27:263-270.

Gabel S, Koncina E, Dorban G, Heurtaux T, Birck C, Glaab E, Michelucci A, Heuschling P, Grandbarbe L (2016) Inflammation promotes a conversion of astrocytes into neural progenitor cells via NF- $\kappa$ B activation. Mol Neurobiol 53:5041-5055.

Garcia ADR, Doan NB, Imura T, Bush TG, Sofroniew MV (2004) GFAP-expressing progenitors are the principal source of constitutive neurogenesis in adult mouse forebrain. Nat Neurosci 7:1233-1241.

Gibert B, Delloye-Bourgeois C, Gattolliat C-H, Meurette O, Le Guernevel S, Fombonne J, Ducarouge B, Lavial F, Bouhallier F, Creveaux M, Negulescu AM, Bénard J, Janoueix-Lerosey I, HarelBellan A, Delattre O, Mehlen P (2014) Regulation by miR181 family of the dependence receptor CDON tumor suppressive activity in neuroblastoma. J Natl Cancer Inst 106:dju318.

Götz M, Sirko S, Beckers J, Irmler M (2015) Reactive astrocytes as neural stem or progenitor cells: in vivo lineage, In vitro potential, and genome-wide expression analysis. Glia 63:1452-1468.

Grinspan JB, Reeves MF, Coulaloglou MJ, Nathanson D, Pleasure D (1996) Re-entry into the cell cycle is required for bFGF-induced oligodendroglial dedifferentiation and survival. J Neurosci Res 46:456-464.

Hegarty SV, Sullivan AM, O'Keeffe GW (2018) Inhibition of miR-181a promotes midbrain neuronal growth through a Smad1/5dependent mechanism: implications for Parkinson's disease. Neuronal Signal 2:NS20170181.

Horn M, Schlote W (1992) Delayed neuronal death and delayed neuronal recovery in the human brain following global ischemia. Acta Neuropathol 85:79-87.
Imayoshi I, Sakamoto M, Ohtsuka T, Takao K, Miyakawa T, Yamaguchi M, Mori K, Ikeda T, Itohara S, Kageyama R (2008) Roles of continuous neurogenesis in the structural and functional integrity of the adult forebrain. Nat Neurosci 11:1153-1161.

Kirino T (1982) Delayed neuronal death in the gerbil hippocampus following ischemia. Brain Res 239:57-69.

Li L, Clevers H (2010) Coexistence of quiescent and active adult stem cells in mammals. Science 327:542-545.

Li L, Stary CM (2016) Targeting glial mitochondrial function for protection from cerebral ischemia: relevance, mechanisms, and the role of microRNAs. Oxid Med Cell Longev 2016:6032306.

Liu XS, Fan BY, Pan WL, Li C, Levin AM, Wang X, Zhang RL, Zervos TM, Hu J, Zhang XM, Chopp M, Zhang ZG (2017) Identification of miRNomes associated with adult neurogenesis after stroke using Argonaute 2-based RNA sequencing. RNA Biol 14:488-499.

Magnusson JP, Frisén J (2016) Stars from the darkest night: unlocking the neurogenic potential of astrocytes in different brain regions. Development 143:1075-1086.

Magnusson JP, Göritz C, Tatarishvili J, Dias DO, Smith EMK, Lindvall O, Kokaia Z, Frisén J (2014) A latent neurogenic program in astrocytes regulated by notch signaling in the mouse. Science 346:237-241.

Moon J, Xu L, Giffard RG (2013) Inhibition of microRNA-181 reduces forebrain ischemia-induced neuronal loss. J Cereb Blood Flow Metab 33:1976-1982.

Moulaert V, Verbunt JA, van Heugten CM, Wade DT (2009) Cognitive impairments in survivors of out-of-hospital cardiac arrest: a systematic review. Resuscitation 80:297-305.

Ouyang Y-B, Voloboueva LA, Xu L-J, Giffard RG (2007) Selective dysfunction of hippocampal CA1 astrocytes contributes to delayed neuronal damage after transient forebrain ischemia. $\mathrm{J}$ Neurosci 27:4253-4260.

Ouyang Y-B, Lu Y, Yue S, Giffard RG (2012a) miR-181 targets multiple $\mathrm{Bcl}-2$ family members and influences apoptosis and mitochondrial function in astrocytes. Mitochondrion 12:213-219.

Ouyang YB, Lu Y, Yue S, Xu LJ, Xiong XX, White RE, Sun X, Giffard RG (2012b) miR-181 regulates GRP78 and influences outcome from cerebral ischemia in vitro and in vivo. Neurobiol Dis 45:555563.

Ouyang YB, Xu L, Yue S, Liu S, Giffard RG (2014) Neuroprotection by astrocytes in brain ischemia: importance of microRNAs. Neurosci Lett 565:53-58.

Platel JC, Bordey A (2016) The multifaceted subventricular zone astrocyte: from a metabolic and pro-neurogenic role to acting as a neural stem cell. Neuroscience 323:20-28.

Pulsinelli WA, Brierley JB, Plum F (1982) Temporal profile of neuronal damage in a model of transient forebrain ischemia. Ann Neurol 11:491-498.

Ren L, Zhu R, Li X (2016) Silencing miR-181a produces neuroprotection against hippocampus neuron cell apoptosis post-status epilepticus in a rat model and in children with temporal lobe epilepsy. Genet Mol Res 15:gmr15049313.

Robel S, Berninger B, Götz M (2011) The stem cell potential of glia: lessons from reactive gliosis. Nat Rev Neurosci 12:88-104.

Salazar-Colocho P, Lanciego JL, Del Rio J, Frechilla D (2008) Ischemia induces cell proliferation and neurogenesis in the gerbil hippocampus in response to neuronal death. Neurosci Res 61:27-37.

Schmidt W, Reymann KG (2002) Proliferating cells differentiate into neurons in the hippocampal CA1 region of gerbils after global cerebral ischemia. Neurosci Lett 334:153-156.

Shimada IS, LeComte MD, Granger JC, Quinlan NJ, Spees JL (2012) Self-renewal and differentiation of reactive astrocyte-derived neural stem/progenitor cells isolated from the cortical peri-infarct area after stroke. J Neurosci 32:7926-7940.

Simon C, Götz M, Dimou L (2011) Progenitors in the adult cerebral cortex: cell cycle properties and regulation by physiological stimuli and injury. Glia 59:869-881.

Stary CM, Giffard RG (2015) Advances in astrocyte-targeted approaches for stroke therapy: an emerging role for mitochondria and microRNAS. Neurochem Res 40:301-307. 
Stary CM, Xu L, Li L, Sun X, Ouyang Y-B, Xiong X, Zhao J, Giffard RG (2017) Inhibition of miR-181a protects female mice from transient focal cerebral ischemia by targeting astrocyte estrogen receptor- $\alpha$. Mol Cell Neurosci 82:118-125.

Sugawara T, Kawase M, Lewén A, Noshita N, Gasche Y, Fujimura M, Chan PH (2000) Effect of hypotension severity on hippocampal CA1 neurons in a rat global ischemia model. Brain Res 877:281-287.

Sun X, Voloboueva LA, Stary CM, Giffard RG (2015) Physiologically normal 5\% O2 supports neuronal differentiation and resistance to inflammatory injury in neural stem cell cultures. J Neurosci Res 93:1703-1712.

Verwer RWH, Sluiter AA, Balesar RA, Baayen JC, Noske DP, Dirven CMF, Wouda J, van Dam AM, Lucassen PJ, Swaab DF (2007) Mature astrocytes in the adult human neocortex express the early neuronal marker doublecortin. Brain 130:3321-3335.

Voloboueva LA, Lee SW, Emery JF, Palmer TD, Giffard RG (2010) Mitochondrial protection attenuates inflammation-induced impairment of neurogenesis in vitro and in vivo. J Neurosci 30:1224212251.

Xie W, Li M, Xu N, Lv Q, Huang N, He J, Zhang Y (2013a) miR-181a regulates inflammation responses in monocytes and macrophages. PLoS One 8:e58639.
Xie W, Li Z, Li M, Xu N, Zhang Y (2013b) miR-181a and inflammation: miRNA homeostasis response to inflammatory stimuli in vivo. Biochem Biophys Res Commun 430:647-652.

Xu L, Emery JF, Ouyang Y-B, Voloboueva LA, Giffard RG (2010) Astrocyte targeted overexpression of Hsp72 or SOD2 reduces neuronal vulnerability to forebrain ischemia. Glia 58:1042-1049.

Xu L-J, Ouyang Y-B, Xiong X, Stary CM, Giffard RG (2015) Poststroke treatment with miR-181 antagomir reduces injury and improves long-term behavioral recovery in mice after focal cerebral ischemia. Exp Neurol 264:1-7.

Yasui DH, Xu H, Dunaway KW, Lasalle JM, Jin L-W, Maezawa I (2013) MeCP2 modulates gene expression pathways in astrocytes. Mol Autism 4:3.

Yokoyama A, Yang L, Itoh S, Mori K, Tanaka J (2004) Microglia, a potential source of neurons, astrocytes, and oligodendrocytes. Glia 45:96-104.

Zeisel A, Muñoz-Manchado AB, Codeluppi S, Lönnerberg P, Manno GL, Juréus A, Marques S, Munguba H, He L, Betsholtz C, Rolny C, Castelo-Branco G, Hjerling-Leffler J, Linnarsson S (2015) Cell types in the mouse cortex and hippocampus revealed by singlecell RNA-seq. Science 347:1138-1142. 